\title{
ON ESTIMATION OF ELECTROSTATIC CAPACITY ${ }^{1}$
}

\section{P. R. GARABEDIAN AND M. SCHIFFER}

1. Introduction. In this paper we prove, by means of Hadamard's variational formula, several monotonicity theorems for the electrostatic capacity of a solid or a lamina. From these monotonicity theorems follow some well known isoperimetric inequalities for the capacity which are, however, not such strong statements as the monotonicity theorems themselves. A point of interest in the development as we present it here is that, up to this time, it has rarely been found possible to solve extremal problems and obtain isoperimetric inequalities directly using only Hadamard's formula, since the validity of the formula depends on piece-wise smoothness of the boundary of the domain under consideration.

2. Volume and capacity. Let $D$ be a finite 3-dimensional domain including the origin in its interior and bounded by a piece-wise smooth surface $S$. We denote by

$$
G=\frac{1}{r}-\gamma+O(r)
$$

the Green's function of $D$ for Laplace's equation, with a pole at the origin, where $r$ is the distance from the origin. We shall call $\gamma$ the inverse capacity of $D$ with respect to the origin, and we shall denote by $V$ the volume of $D$. Obviously, $\gamma^{-1}$ has the dimension of length.

Let a deformation of $S$ be described by an infinitesimal shift of $S$ through $\delta n$ units along its inner normal $n$; we obtain the well known Hadamard variational formulas [2]

$$
\begin{aligned}
& \delta \gamma=\frac{1}{4 \pi} \iint_{S}\left(\frac{\partial G}{\partial n}\right)^{2} \delta n d \sigma, \\
& \delta V=-\iint_{S} \delta n d \sigma
\end{aligned}
$$

for the corresponding first order changes in $\gamma$ and $V$, where $d \sigma$ is the surface element on $S$. These formulas show that $\gamma$ and $V$ are monotonically decreasing and increasing functionals of the domain $D$, respectively. We shall now consider the behavior of the dimension-

Presented to the Society, September 5, 1953; received by the editors April 20, 1953 and, in revised form, July 8, 1953.

1 Paper written under the sponsorship of the Office of Naval Research. 
less quantity $V \gamma^{3}$.

Let $G_{a}$ be the Green's function with pole at the origin of the sphere $E_{a}$ of radius $a$ about the origin. Let $a$ be so chosen that $E_{a}$ is the largest such sphere contained in $D$, and let $K_{a}$ denote the points, arcs, or surface elements of $S$ which intersect the boundary of $E_{a}$. We consider now a Hadamard shift of $S$ which consists in adding the sphere $E_{a+\delta a}$ to $D$, for small $\delta a>0$. The resulting increment in $V \gamma^{3}$ is, according to (1) and (2),

$$
\delta\left(V \gamma^{3}\right)=-\delta a \iint_{K_{a}}\left[\frac{3 V \gamma^{2}}{4 \pi}\left(\frac{\partial G}{\partial n}\right)^{2}-\gamma^{3}\right] d \sigma .
$$

We wish to show that this increment is negative, and hence that $V \gamma^{3}$ is a decreasing functional for this type of variation.

By the maximum principle, the harmonic function $G-G_{a}$ is nonnegative in $E_{a}$, since it is non-negative on the boundary of $E_{a}$. Since $G-G_{a}$ vanishes on $K_{a}$, we deduce that

$$
\frac{\partial G}{\partial n} \geqq \frac{\partial G_{a}}{\partial n}=\frac{1}{a^{2}}
$$

there. On the other hand, we can compare the volume $V$ and inverse capacity $\gamma$ of $D$ with the corresponding quantities for $E_{a}$ to obtain

$$
V \geqq \frac{4 \pi a^{3}}{3}, \quad \gamma \leqq \frac{1}{a},
$$

since $V$ and $\gamma$ are monotonic in dependence on their domain. Combining (4) and (5), we have on $K_{a}$

$$
\frac{3 V}{4 \pi \gamma}\left(\frac{\partial G}{\partial n}\right)^{2} \geqq 1
$$

whence by (3) we obtain the final conclusion $\delta\left(V \gamma^{3}\right) \leqq 0$.

We can now consider, for an arbitrary positive value of $a$, the dimensionless quantity $V \gamma^{3}$ associated with the domain found by adding the sphere $E_{a}$ to $D$. Our discussion proves that this quantity is a nonincreasing function of the radius $a$. For sufficiently small $a>0$, it coincides with the value of $V \gamma^{3}$ for $D$, while for large radii $a$, it reduces to the value $4 \pi / 3$ of $V \gamma^{3}$ for a sphere. Thus a corollary of the monotonic dependence of $V \gamma^{3}$ on the parameter $a$ is the familiar result [4] that $V \gamma^{3}$ is a minimum when $D$ is a sphere about the origin.

By similar calculations, with all the inequalities reversed, we can show that the quantity $V \gamma^{3}$ to be associated with the intersection of 
$D$ and the sphere $E_{a}$ is a nondecreasing function of the radius $a$. This again suffices to demonstrate the extremal character of the sphere.

Formal application of Hadamard's formulas (1) and (2) to the extremal problem $V \gamma^{3}=$ minimum leads to the variational condition

$$
\frac{\partial G}{\partial n}=\text { const. }
$$

Our method shows directly that the sphere is the only surface for which (7) is identically fulfilled. For if $S$ is such a surface, let $E_{a}$ be the largest sphere included in $D$ and let $E_{b}$ denote the smallest sphere containing $D$. By the maximum principle, $G_{a} \leqq G \leqq G_{b}$, whence, computing normal derivatives at points of tangency of the boundaries of $E_{a}$ and $D$ and of $D$ and $E_{b}$, we find

$$
\frac{1}{a^{2}}=\frac{\partial G_{a}}{\partial n} \leqq \frac{\partial G}{\partial n} \leqq \frac{\partial G_{b}}{\partial n}=\frac{1}{b^{2}} .
$$

But, obviously, $a^{2} \leqq b^{2}$, whence $a=b$ and $S$ must be a sphere about the origin.

3. Capacity of laminas. Let now $D$ be an infinite domain bounded by a finite piece-wise smooth lamina $S$ spanned through a curve $C$. We denote by

$$
G=1-\frac{\Gamma}{r}+o\left(\frac{1}{r^{2}}\right)
$$

the Green's function of $D$ with pole at infinity, where the constant $\Gamma$ is the electrostatic capacity of the lamina $S$. For an inner normal shift $\delta n$ of $S$, the Poincare-Hadamard formula $[2 ; 3]$ for the variation of $\Gamma$ is

$$
\delta \Gamma=\frac{1}{4 \pi} \iint_{S}\left(\frac{\partial G}{\partial n}\right)^{2} \delta n d \sigma,
$$

where the integration is to be carried out over both sides of $S$.

Suppose coordinates are chosen so that the $(x, y)$-plane is tangent to $S$ at the origin, and suppose further that no point of $S$ lies in the halfspace $z>0$ and that $C$ lies in the half-space $z<0$. For small $\epsilon>0$, we make a Hadamard shift of $S$ which consists in replacing those elements of $S$ lying in the region $z>-\epsilon$ by the portions of the plane $z$ $=-\epsilon$ cut out by $S$. We denote by $K$ the intersection of $S$ with the plane $z=0$ and on $K$ we denote by $\partial G / \partial z^{+}$the inner normal derivative of $G$ with respect to the region $z>0$, while we denote by $\partial G / \partial z^{-}$ 
the inner normal derivative of $G$ with respect to the portion of $D$ in the half-space $z<0$. By (9), we find for the first order shift in $\Gamma$ under such a plane variation

$$
\delta \Gamma=-\frac{\epsilon}{4 \pi} \iint_{K}\left[\left(\frac{\partial G}{\partial z^{+}}\right)^{2}-\left(\frac{\partial G}{\partial z^{-}}\right)^{2}\right] d \sigma,
$$

where the integration is, of course, carried out just once over the plane region $K$.

We wish to prove that $\delta \Gamma \leqq 0$, and that consequently the capacity $\Gamma$ decreases steadily as we push $S$ back into the convex hull of $C$ with tangent planes. It suffices for this purpose, by (10), to show that on $K$ the inequality

$$
\frac{\partial G}{\partial z^{+}} \geqq \frac{\partial G}{\partial z^{-}}
$$

is fulfilled. We consider, therefore, the harmonic function $G(x, y, z)$ $-G(x, y,-z)$ in that portion $B$ of the half-space $z>0$ where the second term is defined. On those portions of the plane $z=0$ which bound $B$, we have, of course, $G(x, y, z)=G(x, y,-z)$, whereas on the remainder of the boundary of $B$ we find $G(x, y, z)>0$ and $G(x, y,-z)$ $=0$. Hence, by the maximum principle

$$
G(x, y, z)-G(x, y,-z) \geqq 0
$$

in $B$. On $K$ we have the boundary conditions $G(x, y, z)=G(x, y,-z)$ $=0$, whence differentiation with respect to $z$ there yields

$$
\frac{\partial G(x, y, z)}{\partial z}-\frac{\partial G(x, y,-z)}{\partial z} \geqq 0,
$$

which is precisely the desired inequality (11), since both derivatives are non-negative.

These calculations show that whenever $S$ protrudes from the convex hull of the boundary curve $C$, a continuous Hadamard variation of $S$ which consists in replacing those portions of $S$ located on the far side of a plane moving toward the convex hull of $C$ by sections of the moving plane itself will monotonically diminish the capacity $\Gamma$. It follows that, in particular, when $S$ is the surface of least capacity which can be spanned through $C$, then $S$ must lie within the convex hull of $C[1]$. The same conclusion can be drawn in like manner for the logarithmic capacity of a plane arc, and in this case we obtain a new demonstration of the familiar theorem [4] that the curve of minimum logarithmic capacity joining two given points in the plane 
is a line segment. Our result is actually stronger than this theorem, since it asserts the monotonically decreasing character of the capacity of an arbitrary arc which is continuously narrowed down between two parallel lines which approach the extremal segment.

In a similar way, extremal properties of other physical quantities can be derived. For example, it can easily be shown that the axially symmetric irrotational flow of an incompressible fluid past a lamina spanned through a given circle perpendicular to the direction of the flow gives rise to the smallest possible value of the virtual mass when the lamina in question reduces to a plane disk.

Finally, the assertion (11) about the charge distribution on two opposite sides of a lamina is in itself of iterest. We can state, by (11), that for a convex lamina, the charge on the outer surface is always greater than the charge on the inner surface.

This result can be generalized in several directions by the use of the Kelvin transformation in place of the ordinary reflection upon which we based formula (11). Consider, for example, a lamina $S$ enclosed in the sphere $E_{a}$ of radius $a$ about the origin and touching the boundary of $E_{a}$ at the point $(0,0, a)$. The harmonic function.

$$
H(x, y, z)=G(x, y, z)-\left(1-\frac{a}{r}\right)-\frac{a}{r} G\left(\frac{a^{2} x}{r^{2}}, \frac{a^{2} y}{r^{2}}, \frac{a^{2} z}{r^{2}}\right)
$$

is regular and non-negative in a certain subdomain of the exterior of $E_{a}$ bounded in part by the inverse image of $S$ with respect to the sphere of radius $a$. At the boundary point $(0,0, a), H$ vanishes, and hence

$$
\frac{\partial H}{\partial z} \geqq 0
$$

there. This leads to the inequality

$$
\frac{\partial G}{\partial n^{+}}-\frac{\partial G}{\partial n^{-}} \geqq \frac{1}{a},
$$

where $n^{+}$and $n^{-}$denote, respectively, the outer and inner normals relative to $E_{a}$ at the point $(0,0, a)$. If $S$ lies outside a sphere of radius $b \leqq a$ lying within $E_{a}$ and touching $S$ at the point $(0,0, a)$, we obtain there in an analogous manner

$$
\frac{1}{b} \geqq \frac{\partial G}{\partial n^{+}}-\frac{\partial G}{\partial n^{-}} .
$$

These interesting estimates of the difference of charge densities on the 
two sides of the lamina $S$ show, in particular, that when $S$ lies on a sphere of radius $a$, the charge densities differ by the constant amount $1 / a$.

\section{REFERENCES}

1. G. C. Evans, Continua of minimum capacity, Bull. Amer. Math. Soc. vol. 47 (1941) pp. 717-773.

2. P. R. Garabedian and M. Schiffer, Convexity of domain functionals, Journal d'Analyse Mathématique vol. 2 (1952-53) pp. 281-368.

3. H. Poincaré, Figures d'équilibre d'une masse fluide, Paris, 1902.

4. G. P6lya and G. Szegö, Isoperimetric inequalities of mathematical physics, Princeton, 1951.

STANFORD UNIVERSITY 